\title{
ANÁLISE DE POSTAGENS SOBRE SUICÍDIO E COMUNIDADE LGBTQ NO TWITTER*
}

ANALYSIS OF SUICIDE AND LGBTQ COMMUNITY POSTS ON TWITTER

Recibido: 29 de octubre de 2019 | Aceptado: 23 de diciembre de 2019

\author{
JONAS GABRIEL PESTANA GRADIM 1, ALINE CONCEIÇÃO SILVA 2, \\ CAMILA CORRÊA MATIAS PEREIRA 3 , KELLY GRAZIANI GIACCHERO VEDANA 4
}

\author{
1., 2., 3., 4. UNIVERSIDADE DE SÃO PAULO, São Paulo, Brasil
}

\begin{abstract}
Background: Suicide is a multi-causal phenomenon, complex and with diverse manifestations according to groups, socio-demographic and cultural aspects. Individuals who do not identify themselves as cisgender or heter osexual have an increased chance of manifesting suicidal behavior. Purpose: The purpose of this study was to analyze LGBTQ community related posts and suicide on Twitter. Method: This is an exploratory, retrospective study with quantitative approach with analysis of 498 posts in Portuguese on LGBT population and suicide. Postings were collected through screen capture, transcribed, coded and then analyzed in the SAS (Statistical Analysis System) version 9.2. Descriptive statistics, association tests and multiple logistic regression were used, with a level of significance of 5\%. Results: Predominant tweets of men, written in second or third person, without judgment to the LGBT community, without incentive to suicide and without expression of suicidal behavior. Majority did not mention suicide method or pact and did not expose groups suffering discrimination. There was low involvement with posts. Criticism of the LGBTQ community was more expressed by men. Association between first-person speech and suicidal behavior. Written posts in first person were more likely to receive likes and comments and postings of response type and had increased chances of being commented. Conclusions: The results of the study address the precautions for use and the possibility of directing the development of online strategies and scientific studies aimed at awareness and prevention of suicidal behavior.
\end{abstract}

KEY WORDS: Sexual and gender minorities, internet, mental health, nursing

\section{CÓMO CITAR / HOW TO CITE}

Pestana Gradim, J. G., Conceição Silva, A., Matias Pereira, C. C., \& Giacchero Vedana, K. G. (2019). Análise de postagens sobre suicídio e comunidade LGBTQ no Twitter. Salud \& Sociedad, 10(3), 286-294. doi: 10.22199/issn.0718-7475-2019-03-018

\footnotetext{
1. Enfermeiro pela Escola de Enfermagem de Ribeirão Preto da Univer sidade de São Paulo EERP-USP, Ribeirão Preto, SP, Bra sil.

E-mail: jonasgpgradim@hotmail.com, ORCID: 0000-0002-5341-1570

2. Doutoranda em Enfermagem Psiquiátrica e Ciências Humanas da Escola de Enfermagem de Ribeirão Preto da Universidade de São Paulo EERP-USP, Ribeirão Preto, SP, Brasil.

E-mail: csilvaaline@hotmail.com, ORCID: 0000-0001-5843-2517

3. Doutoranda em Enfermagem Psiquiátrica e Ciências Humanas da Escola de Enfermagem de Ribeirão Preto da Univer sidade de São Paulo EERP-USP, Ribeirão Preto, SP, Brasil. E-mail:milamatias@hotmail.com,0RCID:0000-0001-6910-4148

4. Professora Doutora do Departamento de Enfermagem Psiquiátrica e Ciências Humanas da Escola de Enfermagem de Ribeirão Preto da Univer sidade de São Paulo EERP-USP, Ribeirão Preto, SP, Brasil. E-mail: kellygiacchero@eerp.usp.br, 0RCID: 0000-0001-7363-2429

* 0 presente trabalho foi realizado com apoio da Fundação de Amparo à Pesquisa do Estado de São Paulo - Brasil (FAPESP) - Projeto Regular - Processo 2017/24024-7 e pela Coordenação de Aperfeiçoamento de Pessoal de Nível Superior - Bra sil (CAPES) - Código de Financiamento 001.
} 
O comportamento suicida engloba espetro de condições com diferente intensidade na intenção de morrer e letalidade (Turecki \& Brent, 2017). Configura-se como grave problema de saúde pública sendo globalmente mais de 804.000 mortes por ano, uma morte a cada 40 segundos e dez vezes mais tentativas que 0 número de mortes. Se configura como segunda causa de morte entre jovens de 15 a 29 anos de idade (Organización Panamericana de la Salud, 2014).

O suicídio é fenômeno multicausal, complexo e que se diversifica conforme grupos, aspectos sócio demográficos e culturais (Organización Panamericana de la Salud, 2014). Para a prevenção do suicídio é importante compreender fatores e mecanismos envolvidos no risco suicida, desenvolver estratégias eficazes para rastrear e acompanhar indivíduos vulneráveis e desenvolver ações que atendam às necessidades de grupos com características especificas associadas a maior vulnerabilidade.

Indivíduos que não se identificam como cisgênero ou como heterossexuais possuem chances aumentadas de manifestar 0 comportamento suicida (ideação, plano, tentativa, morte). Evidências indicam que a população Lésbicas, Gays, Bissexuais, Transexuais e Queer (LGBTQ+) está em risco substancialmente maior de suicídio (Hatzenbuehler, Birkett, Wagenen, \& Meyer, 2014; Hottes, Bogaert, Rhodes, Brennan, \& Gesink, 2016). Dentre os fatores associados ao risco, encontram-se problemas de relacionamento, de aceitação da homossexualidade, homofobia, discriminação institucional, isolamento social e alienação (Wang, Plöderl, Häusermann, \& Weiss, 2015). Pesquisadores apontam a relutância de jovens LGBTQ+ em procurar ajuda em serviços de saúde mental devido, principalmente, à vergonha em "transgredir normas e padrões de normalidade heterossexuais" (Mcdermott, Roen, \& Scourfield, 2008).
Com a facilidade de acesso à internet, jovens LGBTQ+ buscam por suporte online relacionado a comportamentos auto infligidos, clarificação sobre gênero e sexualidade, superação de homofobia e angústia emocional (Mcdermott, 2014; Mcdermott \& Roen, 2012). Pesquisas destacam a inserção de grupos antes marginalizados na internet e advertem sobre as consequências do uso. A internet permite espaço de apoio, informação e interação mas também pode operar de forma inversa aumentando riscos para jovens vulneráveis (Dinamarco, 2011).

Para trabalhar a prevenção do comportamento suicida é necessário compreender as experiências de diferentes grupos e em diferentes contextos. A transformação das necessidades sociais exige do cuidado em saúde constante movimentação para abarcar as complexidades e novas demandas sociais. Assim, se faz necessário a inserção do contexto online no cuidado em saúde mental. Dessa forma o objetivo deste trabalho é analisar postagens relacionadas a comunidade LGBTQ+ e suicídio no Twitter.

\section{MÉTODO}

\section{Desenho do estudo}

Estudo de escopo descritivo e exploratório com abordagem quantitativa para permitir uma abordagem inicial do conhecimento científico sobre postagens com conteúdo sobre suicídio e comunidade LGBT no Twitter e indicar recomendações técnicas e de design para estudos adicionais.

\section{Cenário}

O Twitter foi fundado em 2006 na Califórnia (EUA) e possibilita que postagens curtas tenham repercussão rápida e elevada. $A$ depender da escolha do usuário, as informações do perfil e publicações podem ser de acesso privado (que requer conta e está sob controle do usuário) ou público (acessivel para qualquer pessoa) (Twitter, 2019). 


\section{Procedimento}

Foi realizada busca de postagens com os termos "suicídio" e "LGBT" no campo de "busca avançada" do Twitter e foram usados filtros para seleção de postagens em língua portuguesa. A coleta de dados foi realizada no dia 12 de outubro de 2017 e foram coletados os tweets postados até as $17 \mathrm{~h} 20 \mathrm{~min}$, horário em que foi encerrada a coleta.

As postagens foram coletadas por meio de captura de telas e posteriormente transcritas em documento editável, revisadas para correção de erros e identificadas por número correspondente à ordem em que foram encontradas.

\section{Critérios de Seleção}

Foram elegíveis todas as postagens identificadas na estratégia de busca com conteúdo em forma de texto que pudesse ser analisado de forma independente de imagens, vídeos ou links vinculados às postagens. Um total de 544 tweets foram identificados pela estratégia de busca, destes, 46 foram excluídos, por não atenderem os critérios de seleção e a amostra do estudo foi composta por 498 tweets.

\section{Aspectos éticos}

O estudo seguiu as recomendações da Resolução $n^{0} 466$, de 12 de dezembro de 2012 do Conselho Nacional de Saúde e a Declaração de Helsinki (Associação Médica Mundial, 1964; Brasil, 2012). Foram analisados apenas os dados públicos, de livre acesso. Foram preservados 0 anonimato dos usuários do Twitter em todas as etapas da pesquisa, bem como os princípios de dignidade humana, autonomia, proteção, segurança, maximização dos benefícios e minimização de danos, respeito pelas pessoas, justiça e beneficência. Variáveis
As características das postagens foram codificadas de acordo com um formulário elaborado por especialistas. Foram consideradas as seguintes variáveis: sexo do autor da postagem (feminino; masculino; inconclusivo - quando não era possível identificar o sexo do autor), tipo de postagem (tweet - publicação produzida pelo autor; retweet - compartilhamento de uma publicação; resposta a uma publicação prévia); curtidas (sim ou não; quantidade), compartilhamentos (sim ou não; quantidade), comentários (sim ou não; quantidade).

Discurso em primeira pessoa (sim ou não); julgamento/crítica à comunidade LGBT (sim ou não); expressão de comportamento suicida do autor da postagem (sim ou não); menção a método para o suicídio (sim ou não); menção a pacto suicida (sim ou não); incentivo explícito ao suicídio (sim ou não); menção a grupos alvos de discriminação (sim ou não); menção a pessoas específicas (sim ou não); menção a entidades, instituições ou organizações específicas (sim ou não).

\section{Análise de dados}

Após a extração dos dados, a fim de organizar o material obtido, foi construído banco de dados no Programa Microsoft Excel 2010. Os dados foram duplamente digitados para evitar possíveis erros, bem como o cruzamento entre a primeira e a segunda digitação para analisar a consistência e veracidade dos dados obtidos. Posteriormente, foram exportados para o programa estatístico SAS (Statistical Analysis System) versão 9.2 e analisados estatisticamente.

Os dados foram analisados por estatística descritiva e posteriormente submetidos a testes de associação (teste qui-quadrado ou o teste exato de Fisher) entre as variáveis de interesse e para verificar se as variáveis de interesse eram preditoras de resposta foram calculados odds ratio através da regressão logística múltipla. Para todos os testes, 0 nível de significância adotado foi de $5 \%$. 


\section{RESULTADOS}

Entre as postagens sobre suicídio e comunidade LGBT predominaram os tweets $(78,51 \%)$, de autores identificados como homens $(43,87 \%)$, escritos em segunda ou terceira pessoa $(73,90 \%)$, sem julgamento ou crítica à comunidade LGBT $(96,59 \%)$, sem incentivo ao suicídio $(97,59 \%)$ e sem expressão de comportamento suicida do autor da postagem $(97,59 \%)$. A maioria das postagens não mencionava método para 0 suicídio $(100 \%)$ ou pacto suicida $(98,79 \%)$ e não expunham grupos que fossem alvo de discriminação $(94,98 \%)$, pessoas específicas $(83,73 \%)$ ou entidades, instituições ou organizações específicas $(74,90 \%)$. No que se refere à interação com as postagens, a maioria não recebeu curtidas $(63,05 \%)$, compartilhamentos $(71,69 \%)$ ou comentários (79,92\%).

\section{Associações entre interações dos usuários e demais características de postagens relacionadas a suicídio e comunidade LGBT}

As postagens sem identificação de sexo tiveram mais chances de serem curtidas $(\mathrm{OR}=2,00 ; \quad \mathrm{IC}=1,09-3,68 ; \quad \mathrm{p}=0,03) \quad \mathrm{e}$ compartilhadas $\quad(\mathrm{OR}=2,97 ; \quad \mathrm{IC}=1,61-5,47$; $p<0,01)$. As postagens escritas em primeira pessoa tiveram mais chance de receberem curtidas $(O R=1,79 ; I C=1,04-3,10 ; p=0,04)$ e comentários $\quad(\mathrm{OR}=2,36 ; \quad \mathrm{IC}=1,22-4,59$; $p=0,01$. As postagens do tipo resposta tiveram chances aumentadas de serem comentadas $\quad(\mathrm{OR}=5,39 ; \quad \mathrm{IC}=2,83-10,26$; $p<0,01$ ) (Tabela 1).

\section{TABELA 1.}

Interação por curtidas, compartilhamentos e comentários e demais características de postagenelacionadas a suicídio e comunidade LGBT ( $\mathrm{n}=498)$ no Twitter.

\begin{tabular}{|c|c|c|c|c|c|c|}
\hline & \multicolumn{2}{|c|}{ Curtidas } & \multicolumn{2}{|c|}{ Compartilhamentos } & \multicolumn{2}{|c|}{ Comentários } \\
\hline & OR $(\mathrm{IC})$ & p valor & $\mathrm{OR}(\mathrm{IC})$ & p valor & OR $(\mathrm{IC})$ & p valor \\
\hline \multicolumn{7}{|l|}{ Sexo } \\
\hline Feminino & $1,35(0,80-2,28)$ & 0,26 & $0,74(0,41-1,33)$ & 0,32 & $0,78(0,40-1,53)$ & 0,48 \\
\hline Masculino & 1,00 & - & 1,00 & - & 1,00 & - \\
\hline Outro & $2,00(1,09-3,68)$ & 0,03 & $2,97(1,61-5,47)$ & $<0,01$ & $1,16(0,50-2,72)$ & 0,73 \\
\hline \multicolumn{7}{|c|}{ Tipo de Postagem } \\
\hline Resposta & $1,61(0,91-2,85)$ & 0,10 & $1,25(0,66-2,36)$ & 0,49 & $5,39(2,83-10,26)$ & $<0,01$ \\
\hline Retweet & * & * & $1,08(0,06-18,99)$ & 0,96 & * & * \\
\hline Tweet & 1,00 & - & 1,00 & - & 1,00 & - \\
\hline \multicolumn{7}{|c|}{ Discurso Primeira Pessoa } \\
\hline Sim & $1,79(1,04-3,10)$ & 0,04 & $1,21(0,65-2,23)$ & 0,55 & $2,36(1,22-4,59)$ & 0,01 \\
\hline Não & 1,00 & - & 1,00 & - & 1,00 & - \\
\hline \multicolumn{7}{|c|}{ Grupos alvo de discriminação } \\
\hline Sim & $1,81(0,57-5,69)$ & 0,31 & $1,43(0,42-4,86)$ & 0,56 & $2,41(0,64-9,16)$ & 0,20 \\
\hline Não & 1,00 & - & 1,00 & - & 1,00 & - \\
\hline \multicolumn{7}{|c|}{ Menciona pessoas específicas } \\
\hline Sim & 1,00 & - & 1,00 & - & 1,00 & - \\
\hline Não & $1,67(0,84-3,31)$ & 0,14 & $0,88(0,45-1,71)$ & 0,70 & $1,62(0,61-4,30)$ & 0,33 \\
\hline \multicolumn{7}{|c|}{ Menciona instituições } \\
\hline Sim & 1,00 & - & 1,00 & - & 1,00 & - \\
\hline Não & $1,65(0,96-2,83)$ & 0,07 & $0,84(0,49-1,44)$ & 0,52 & $0,95(0,47-1,90)$ & 0,87 \\
\hline \multicolumn{7}{|c|}{ Julgamento/crítica à comunidade LGBT } \\
\hline Sim & $2,86(0,87-9,40)$ & 0,08 & $2,02(0,62-6,57)$ & 0,24 & $3,09(0,89-10,74)$ & 0,08 \\
\hline Não & 1,00 & - & 1,00 & - & 1,00 & - \\
\hline \multicolumn{7}{|c|}{ Menciona pacto suicida } \\
\hline Sim & * & * & * & * & * & * \\
\hline Não & 1,00 & - & 1,00 & - & - & - \\
\hline \multicolumn{7}{|c|}{ Comportamento Suicida } \\
\hline Sim & 1,00 & - & 1,00 & - & 1,00 & - \\
\hline Não & $3,34(0,32-35,3)$ & 0,32 & $1,05(0,10-11,05)$ & 0,97 & $0,20(0,01-2,86)$ & 0,24 \\
\hline \multicolumn{7}{|c|}{ Incentivo ao suicídio } \\
\hline Não & * & * & * & - & * & * \\
\hline Sim & 1,00 & - & $1,00\left(^{*}\right)$ & * & 1,00 & - \\
\hline
\end{tabular}


Associações entre conteúdos publicados e

demais características de postagens

relacionadas a suicídio e comunidade LGBT

Foram testadas associações entre os conteúdos publicados e diferentes características das postagens e foram identificadas apenas a associação entre 0 discurso em primeira pessoa e a expressão de comportamento suicida do autor da postagem $(p=0,01)$ (Tabela 2), bem como a associação entre autoria e expressão de julgamento ou críticas à comunidade LGBT, sendo que homens expressaram maior julgamento e autores sem identificação de sexo não apresentavam julgamentos ou críticas nas publicações $(p<0,01)$ (Tabela 3$)$.

\section{TABELA 2.}

Menção a pacto suicida, expressão de comportamento suicida e incentivo ao suicídio e demais características de postagens relacionadas a suicídio e comunidade LGBT $(n=498)$ no Twitter.

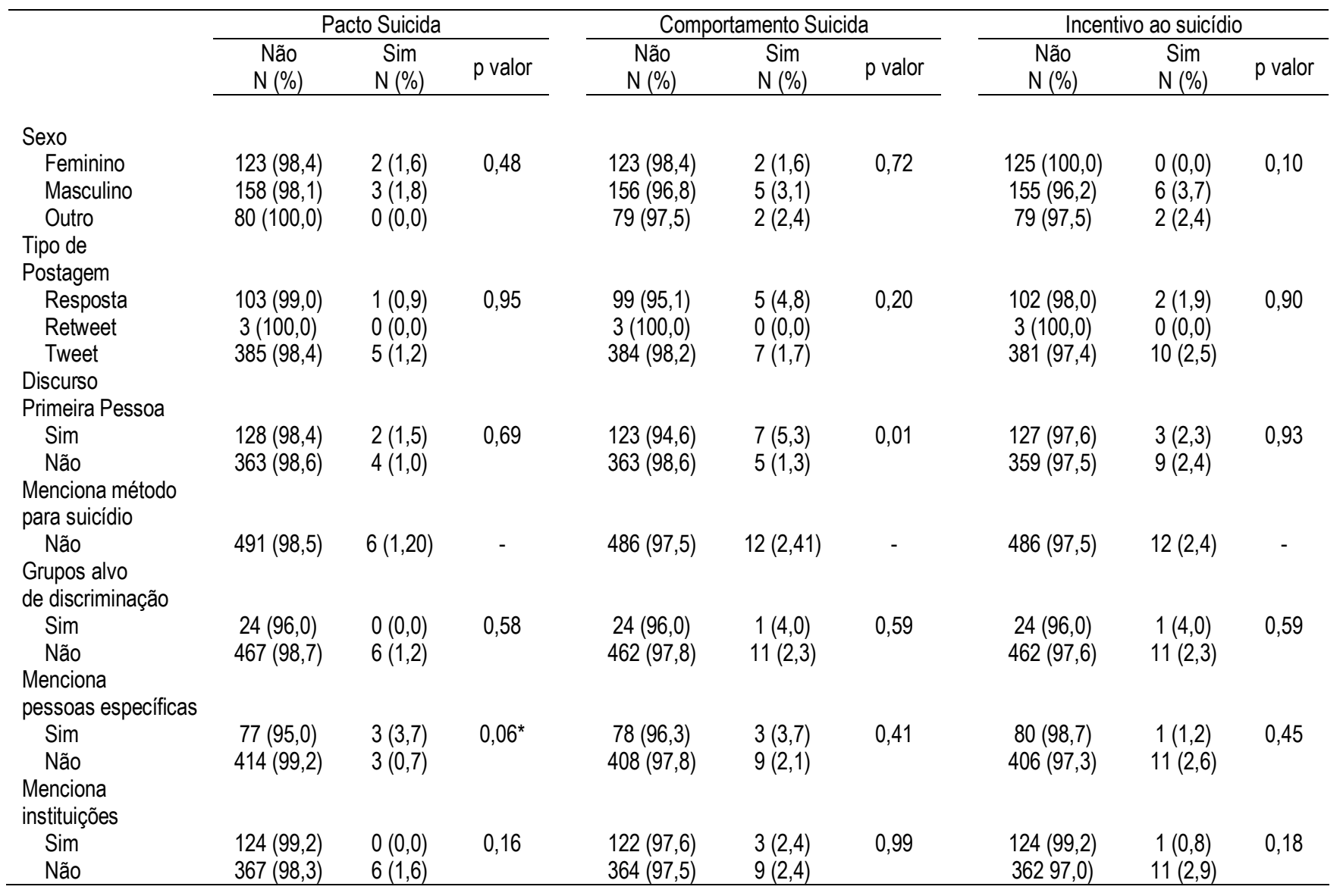

TABELA 3.

Julgamento ou crítica à comunidade LGBT e demais características de postagens relacionadas a suicídio e comunidade LGBT $(n=498)$ no Twitter.

\begin{tabular}{|c|c|c|c|}
\hline & \multicolumn{2}{|c|}{$\begin{array}{l}\text { Julgamento/crítica à } \\
\text { comunidade LGBT }\end{array}$} & \multirow[b]{3}{*}{ p valor } \\
\hline & Sim & Não & \\
\hline & $\mathrm{N}(\%)$ & $N(\%)$ & \\
\hline \multicolumn{4}{|l|}{ Sexo } \\
\hline Feminino & $2(1,6)$ & $123(98,4)$ & $<0,01$ \\
\hline Masculino & $11(6,8)$ & $150(93,1)$ & \\
\hline Outro & $0(0,0)$ & $81(100,0)$ & \\
\hline
\end{tabular}




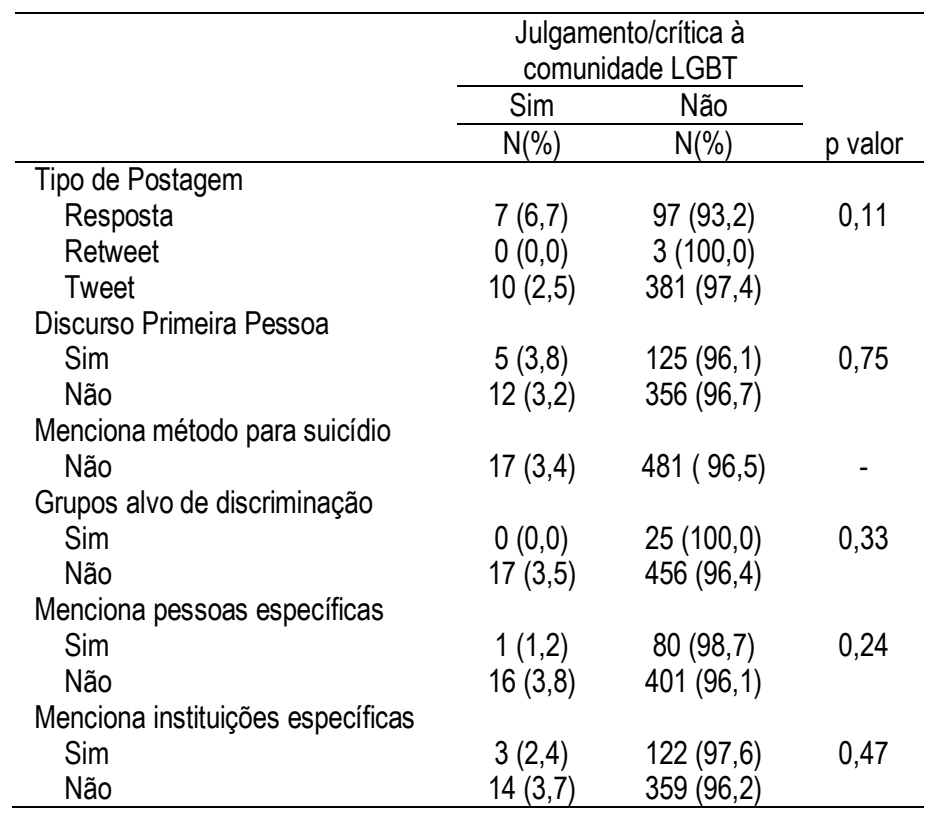

\section{DISCUSSÃO}

Predominaram postagens de autores identificados como homens escritas em segunda ou terceira pessoa. Pesquisas apontam que homens gays são mais propensos a experienciar comportamento suicida e possuem intencionalidade suicida mais forte quando comparados a homens heterossexuais (Hottes et al., 2016). Este pode ser um grupo com necessidades de expressão de necessidades e apoio relacionado ao risco suicida. O predomínio de postagens do tipo tweet demonstra 0 maior interesse ou necessidade dos autores de publicar postagens originais, individuais.

A maioria das postagens não apresentavam julgamento ou crítica à comunidade LGBTQ+ e não expunha grupos discriminados. Tal resultado pode ter associação com a escolha do termo de busca LGBT, considerado mais adequado e respeitoso, comumente utilizado pela própria comunidade, e que se destaca em pesquisas pelo acesso à internet motivado a compreensão de sentimentos ou demanda de apoio e disposição para apoio a outras pessoas LGBTQ (Mcdermott, 2015). As crtíticas à comunidade LGBTQ+ foram mais expressas por homens e menos presentes em postagens de autores sem identificação de sexo. Alguns resultados do presente estudo não puderam ser comparados à literatura devido à falta de estudos semelhantes.

Não foram expostas pessoas específicas ou entidades, instituições ou organizações específicas. Por um lado, esse achado revela a pouca exposição direta de pessoas ou grupos, o que pode ser uma forma de proteção contra o estigma e discursos vexatórios (Lee, Oliffe, Kelly, \& Ferlatte, 2017). Todavia, também pode haver uma lacuna nas publicações sobre recursos e grupos específicos que pudessem ser fonte de pertença, apoio e orientações. A importância do trabalho em rede é fundamental para prevenção do comportamento suicida, conhecer e estreitar diálogo com lideranças do movimento pode colaborar para difundir informações contra estigma e necessidade de apoio profissional conjunto com estratégias de apoio (organizações, linhas de apoio, internet).

A maioria das postagens não mencionava método para o suicídio ou pacto suicida, incentivo suicida ou expressão de comportamento suicida do autor da postagem (Mcdermott, 2015) destaca 0 acesso online da população LGBTQ+ na 
tentativa de compreender ou lidar com 0 sofrimento emocional. Uma das fontes de sofrimento podem ser os atos violentos marcados por homofobia e falta de aceitação. Jovens brasileiros decididos ou questionadores da própria orientação sexual ou identidade de gênero sofrem violência física, psicológica e social. Dados do relatório anual do Grupo Gay da Bahia (2017) mostram que 445 LGBT morreram em 2017, sendo 387 assassinados e 58 suicídios, todos vítimas diretas $\mathrm{e}$ indiretas de homotransfobia (Bahia, 2017).

Postagens autodirigidas (discurso em primeira pessoa) tiveram associação com expressão de comportamento suicida (ideação, tentativa, planificação e morte). A maneira como é expresso o comportamento suicida em ambiente online pode ter duplo poder nocivo, tanto para a pessoa que expressa e consequentemente necessita de apoio qualificado quanto para pessoas vulneráveis expostas ao conteúdo. Pesquisadores alertam para 0 poder amplificador da mídia em casos de efeito contágio ou de imitação e as consequências em pessoas vulneráveis (Westerlund, Hadlaczky, \& Wasserman, 2012).

Nesse estudo, houve um baixo envolvimento com as postagens (curtidas, comentários e compartilhamentos), mas 0 envolvimento mostrou-se associado a características específicas das postagens, os mecanismos envolvidos nesse envolvimento que merecem ser avaliados em outros estudos. As postagens sem identificação de sexo (nas quais o autor não era caracterizado como homem ou de mulher) tiveram mais chances de serem curtidas e compartilhadas. As postagens escritas em primeira pessoa (que podem favorecer a exposição mais direta ou íntima das próprias ideias) tiveram mais chance de receberem curtidas e comentários.

Destaca-se que as postagens do tipo resposta tiveram aproximadamente cinco vezes a chance de serem comentadas quando comparadas aos tweets. Pesquisas apontam que estratégias atuais institucionais sobre prevenção do suicídio em ambiente online se constituem de monólogos e possuem pouca repercussão, contrapondo com alta repercussão percebida pela grande interação de postagens privadas (Westerlund et al., 2012). As abordagens que envolvam respostas aos usuários podem ser avaliadas como recursos associados a maior adesão a estratégias de apoio que envolvam ambientes virtuais.

O estudo possui limitações relacionadas a ausência de técnicas de redução baseadas em critérios empíricos (técnicas de integração, mineração e limpeza de dados, discretização, numeração); limitações das inferências obtidas pela análise dos dados (sem a utilização de técnicas de dimensionalidade e visualização ou outras formas de análise adicionais). Ademais, 0 estudo empregou uma única fonte de coleta de dados e uma única estratégia de busca, e utilizou a captura de tela (que não permite análise de material audiovisual, links e interações, análise de perfis de usuário de forma individualizada e longitudinal). A despeito dessas limitações, 0 presente estudo aponta recomendações importantes para 0 avanço na pesquisa sobre 0 assunto.

\section{Conclusões}

Este é um estudo de escopo exploratório e descritivo no qual foram analisadas postagens relacionadas a comunidade LGBT e comportamento suicida no Twitter. Predominaram postagens de autores identificados como homens, do tipo tweet escritos em segunda ou terceira pessoa. Homens expressaram maior julgamento e autores sem identificação de sexo não apresentavam julgamentos ou críticas nas publicações. Postagens autodirigidas tiveram associação com expressão de comportamento suicida. As postagens sem identificação de sexo tiveram mais chances de serem curtidas e compartilhadas. As postagens escritas em primeira pessoa tiveram mais chance de receberem curtidas 
e comentários. As postagens do tipo resposta tiveram chances aumentadas de serem comentadas.

Os resultados apresentados são relevantes por se apresentarem como uma abordagem inicial e específica de tweets sobre suicídio e população LGBTQ. Os resultados indicam recomendações técnicas e de design relevantes para o planejamento de estudos relacionados à prevenção do comportamento suicida com enfoque na população LGBTQ.

\section{REFERÊNCIAS}

Associação Médica Mundial. (1964). Declaração de Helsique da Associação Médica Mundial (WMA).

Bahia, G. G. da. (2017). Mortes violentas de LGBT no Brasil - Relatório 2017.

Brasil. Resolução no466, de 12 de Dezembro de 2012 (2012).

Dinamarco, A. V. (2011). Análise exploratória sobre o sintoma de automutilação praticada com objetos cortantes e/ou perfurantes, através de relatos expostos na internet por um grupo brasileiro que se define como praticante de automutilação TT - Exploratory analysis on the sympto, 182. http://www.teses.usp.br/teses/disponive is/47/47133/tde-06092011162704/publico/dinamarco_me.pdf

Hatzenbuehler, M. L., Birkett, M., Wagenen, A. Van, \& Meyer, I. H. (2014). Protective school climates and reduced risk for suicide ideation in sexual minority youths. American Journal of Publica Health, 104(2), 279-286. https://doi.org/10.2105/AJPH.2013.301 508

Hottes, T. S., Bogaert, L., Rhodes, A. E., Brennan, D. J., \& Gesink, D. (2016). Lifetime prevalence of suicide attempts among sexual minority adults by study sampling strategies: A systematic review and meta-analysis. American Journal of Public Health Research, 106(5), $1-12$. https://doi.org/10.2105/AJPH.2016.303
088

Lee, C., Oliffe, J. L., Kelly, M. T., \& Ferlatte, O. (2017). Depression and suicidality in gay men: Implications for health care providers. American Journal of Men's Health, 11(4), 910-919. https://doi.org/10.1177/1557988316685 492

Mcdermott, E. (2014). Asking for help online : Lesbian , gay , bisexual and articulating the 'failed' self. Health, 19(6), 561-577. https://doi.org/10.1177/1363459314557 967

Mcdermott, E., \& Roen, K. (2012). Youth on the virtual edge: Researching marginalized sexualities and genders online. Qualitative Health Research, 22(4), 560-570. https://doi.org/10.1177/1049732311425 052

Mcdermott, E., Roen, K., \& Scourfield, J. (2008). Avoiding shame: Young LGBT people, homophobia and selfdestructive behaviours. Culture Health \& Sexuality, 10(8), 815-829. https://doi.org/10.1080/1369105080238 0974

Organización Panamericana de la Salud. (2014). Mortalidad por Suicidio en las Américas.

http://www.paho.org/pan/index.php?opti on=com_docman\&task=doc_details\&gi $\mathrm{d}=253$ \&ltemid=224.

Turecki, G., \& Brent, D. A. (2017). Suicide and suicidal behaviour. Lancet, 387(10024), 1227-1239. https://doi.org/10.1016/S01406736(15)00234-2.

Twitter. (2019). Twitter 2019.pdf.

Vandenbroucke, J. P., Elm, E. Von, Altman, D. G., Gøtzsche, P. C., Mulrow, C. D., Pocock, S. J., ... Initiative, S. (2014). Strengthening the reporting of observational studies in epidemiology ( STROBE ): Explanation and elaboration. International Journal of Surgery, 12(12), 1500-1524. https://doi.org/10.1016/j.ijsu.2014.07.01 4.

Wang, J., Plöderl, M., Häusermann, M., \& 
Weiss, M. G. (2015). Understanding

suicide attempts among gay men from

their self-perceived sauses. The Journal

of Nervous and Mental Disease, 203(7), 499-506.

https://doi.org/10.1097/NMD.00000000 00000319.

Westerlund, M., Hadlaczky, G., \& Wasserman, D. (2012). The representation of suicide on the internet:

Todos los trabajos publicados en Revista Salud \& Sociedad (ISSNe:0718-7475) están sujetos a una licencia Creative Commons Reconocimiento 4.0 Internacional

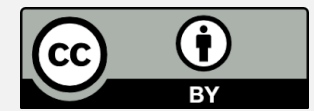

\section{RESUMO}

Antecedentes: 0 suicídio é fenômeno multicausal, complexo e que se diversifica conforme grupos, aspectos sócio demográficos e culturais. Indivíduos que não se identificam como cisgênero ou como heterossexuais possuem chances aumentadas de manifestar 0 comportamento suicida. Objetivo: 0 objetivo deste estudo é analisar postagens relacionadas a comunidade LGBTQ+ e suicídio no Twitter. Metodo: Estudo de escopo descritivo e exploratório, retrospectivo com abordagem quantitativa com análise de 498 postagens em português sobre população LGBT e suicídio. As postagens foram coletadas por meio de captura de tela, transcritas, codificadas. Analisadas no SAS (Statistical Analysis System) versão 9.2. Foram utilizadas estatística descritiva, testes de associação e regressão logística múltipla com nível de significância adotado foi de 5\%. Resultados: Predominaram tweets de homens, escritos em segunda ou terceira pessoa, sem julgamento à comunidade LGBT, sem incentivo ao suicídio e sem expressão de comportamento suicida. Maioria não mencionava método ou pacto suicida e não expunham grupos sofrendo discriminação. Houve baixo envolvimento com as postagens. As críticas à comunidade LGBTQ+ for am mais expressas por homens. Postagens escritas em primeira pessoa tiveram mais chance de receberem curtidas e comentários e postagens do tipo resposta tiveram chances aumentadas de serem comentadas. Conclusões: 0 estudo aborda recomendações a serem consideradas na elaboração de estratégias e estudos científicos voltados para a prevenção de comportamento suicida em ambientes virtuais.

PALAVRAS-CHAVE: Minorias sexuais e de gênero, internet, saúde mental, enfermagem

\section{RESUMEN}

Antecedentes: El suicidio es un fenómeno multicausal, complejo y que se manifiesta de manera diversa según grupos, aspectos sociodemográficos y culturales. Las personas que no se identifican como cisgénero o heterosexual tienen una mayor probabilidad de manifestar un comportamiento suicida. Objetivo: El objetivo de este estudio es analizar posturas relacionadas a la comunidad LGBTQ y suicidio en Twitter. Métodos: Un estudio exploratorio, retrospectivo, con un enfoque cuantitativo con el análisis de 498 mensajes en portugués sobre la población LGBT y el suicidio. Método: Las entradas fueron recolectadas por medio de captura de pantalla, transcritas, y codificadas. Luego, las analizamos utilizando SAS (Statistical Analysis System) versión 9.2. Utilizamos estadísticas descriptivas, pruebas de asociación y regresión logística múltiple con nivel de significancia adoptado de 5\%. Resultados: Predominaron tweets de hombres, escritos en segunda 0 tercera persona, sin juicio a la comunidad LGBT, sin incentivo al suicidio y sin expresión de comportamiento suicida. La mayoría no mencionaba método o pacto suicida y no exponían grupos que sufren discriminación. Hubo poca participación en las entradas. Las críticas a la comunidad LGBTQ fueron más expresadas por hombres. Asociación entre el discurso en primera persona y expresión de comportamiento suicida. Las publicaciones escritas en primera persona tuvieron más posibilidades de recibir me gusta, comentarios y posturas del tipo respuesta, tuvieron más posibilidades de recibir comentarios. Conclusiones: Los resultados del estudio abordan las precauciones de uso y la posibilidad de dirigir el desarrollo de estrategias en línea y estudios científicos dirigidos a la concienciación y la prevención del comportamiento suicida.

PALABRAS CLAVE: Minorías sexuales y de género, internet, salud mental, enfermería 